\title{
Airway Hyperresponsiveness in Asthma: A Problem of Limited Smooth Muscle Relaxation with Inspiration
}

Gwen Skloot, ${ }^{*}$ Solbert Permutt, ${ }^{\star}$ and Alkis Togias ${ }^{\star *}$

Department of Medicine, * Division of Pulmonary and Critical Care Medicine and ${ }^{\ddagger}$ Division of Clinical Immunology, The Johns Hopkins Asthma and Allergy Center, Baltimore, Maryland 21224-6801

\begin{abstract}
We hypothesized that hyperresponsiveness in asthma is caused by an impairment in the ability of inspiration to stretch airway smooth muscle. If the hypothesis was correct, we reasoned that the sensitivity to inhaled methacholine in normal and asthmatic subjects should be the same if the challenge was carried out under conditions where deep inspirations were prohibited. 10 asthmatic and 10 normal subjects received increasing concentrations of inhaled methacholine under conditions where forced expirations from a normal end-tidal inspiration were performed. When no deep inspirations were allowed, the response to methacholine was similar in the normal and asthmatic subjects, compatible with the hypothesis we propose. Completely contrary to our expectations, however, was the marked responsivity to methacholine that remained in the normal subjects after deep breaths were initiated. 6 of the 10 normal subjects had $>20 \%$ reduction in forced expiratory volume in one second $\left(\right.$ FEV $_{1}$ ) at doses of methacholine $<8 \mathrm{mg} / \mathrm{ml}$, whereas there was $<15 \%$ reduction with $75 \mathrm{mg} / \mathrm{ml}$ during routine challenge. The ability of normal subjects to develop asthmatic responses when the modulating effects of increases in lung volume was voluntarily suppressed suggests that an intrinsic impairment of the ability of inspiration to stretch airway smooth muscle is a major feature of asthma. (J. Clin. Invest. 1995. 96:2393-2403.) Key words: asthma • airway hyperresponsiveness $\bullet$ airway smooth muscle $\bullet$ pulmonary mechanics
\end{abstract}

\section{Introduction}

In 1961, Nadel and Tierney showed that while a deep inspiration did not alter airway resistance measured at functional residual capacity in the control state, it always reduced it for 1-2 min when bronchoconstriction was present (1). In 1981, Fish et al.

Address correspondence to Alkis Togias, The Johns Hopkins Asthma and Allergy Center, 5501 Hopkins Bayview Circle, Baltimore, MD 21224. Phone: 410-550-2189; FAX: 410-550-2193.

Received for publication 23 January 1995 and accepted in revised form 24 July 1995

1. Abbreviations used in this paper: $\tau$, time constant; DI, deep inspiration; $\mathrm{FEV}_{1}$, forced expiratory volume in one second; FVC, forced vital capacity; IVC, inspiratory vital capacity; Mch, methacholine; MMEF, mid maximal expiratory flow; $\mathrm{PC}$, provocative concentration; RV, residual volume; TLC, total lung capacity.

J. Clin. Invest.

(C) The American Society for Clinical Investigation, Inc.

$0021-9738 / 95 / 11 / 2393 / 11 \$ 2.00$

Volume 96, November 1995, 2393-2403 confirmed the findings of Nadel and Tierney in nonasthmatic subjects following a challenge with methacholine (Mch), ${ }^{1}$ but the effect of a deep inspiration was much less in asthmatic subjects (2). Fish et al. felt that there was an intrinsic means of regulating bronchomotor tone by active changes in lung volume in normal subjects, but the mechanism was somehow impaired in asthma. They suggested, "that airway hyperreactivity in asthma is perhaps less a reflection of enhanced end-organ responsiveness than a reflection of this impaired capacity." The observations of Nadel and Tierney and Fish et al. in normal subjects could be explained by the stretch of constricted airway smooth muscle causing a transient reduction in smooth muscle tone (3). Fish et al. suggested that the major problem in asthma might be an impairment in the ability of inspiration to stretch the airway smooth muscle $(3,4)$. This was in keeping with the findings of Colebatch et al. in 1973 (5) who observed that there was a much smaller decrease in pulmonary resistance with lung inflation in asthmatic than healthy subjects; and they suggested that this was due to an "increased rigidity of the airways." In 1974, Green and Mead speculated that "perhaps in patients with asthma maximal inspiration is unable to dilate the airways completely,"' (6).

If the hypothesis of Fish et al. were correct, normal subjects should respond like asthmatic subjects to airway smooth muscle constrictors if the challenge could be carried out under conditions where the bronchodilating effects of deep inspiration in the normal subjects could be suppressed. This is a corollary of the hypothesis of Fish et al., and the testing of this corollary was the aim of the present study.

We measured the response to increasing concentrations of Mch in both normal and asthmatic subjects in a protocol where deep breaths were prohibited so that lung volumes never increased above the volume at a normal end-tidal inspiration. Under these conditions the response to increasing concentrations of Mch was similar in the normal and asthmatic subjects, compatible with the corollary hypothesis. What was especially surprising and provocative was that the effect of Mch in the normal subjects was markedly exaggerated even after deep breaths, as in asthma, after the prolonged inhibition of deep breaths during the challenge; whereas, we had expected a completely normal response once the deep breaths were initiated in the normal subjects. The ability of normal subjects to develop asthmatic responses when the modulating effect of increases in lung volume was voluntarily suppressed for prolonged periods of time suggests that the intrinsic impairment of the same modulating effect in asthma may be a major feature of the disease.

\section{Methods}

This study was approved by the Institutional Review Board of the Johns Hopkins Bayview Medical Center. Informed, written consent was obtained from each subject before enrollment. 
Table I. Demogrpahics and Baseline Characteristics of Asthmatic and Normal Subjects

\begin{tabular}{|c|c|c|c|c|c|c|c|c|}
\hline Subject & Age & Gender & $\begin{array}{l}\text { Skin test } \\
\text { reactivity }\end{array}$ & $\begin{array}{l}\mathrm{FEV}_{1} \% \\
\text { predicted }\end{array}$ & $\begin{array}{l}\text { FVC \% } \\
\text { predicted }\end{array}$ & $\mathrm{FEV}_{1} / \mathrm{FVC}$ & Mch $\mathrm{PC}_{20} *$ & Medications \\
\hline \multicolumn{9}{|c|}{ Asthmatics } \\
\hline 1 & 37 & $\mathbf{M}$ & + & 69 & 70 & 0.76 & 0.13 & $\operatorname{prn} \beta$-agonist, prn theophylline \\
\hline 2 & 26 & $\mathbf{M}$ & + & 49 & 65 & 0.65 & 0.07 & $\beta$-agonist \\
\hline 3 & 39 & $\mathrm{~F}$ & + & 81 & 87 & 0.77 & 0.21 & prn $\beta$-agonist \\
\hline 4 & 32 & $\mathbf{M}$ & + & 96 & 102 & 0.80 & 0.21 & - \\
\hline 5 & 24 & $\mathbf{M}$ & + & 48 & 81 & 0.51 & 0.02 & $\beta$-agonist \\
\hline 6 & 30 & F & + & 74 & 92 & 0.69 & 0.14 & $\beta$-agonist, theophylline \\
\hline 7 & 34 & $\mathbf{M}$ & + & 62 & 84 & 0.62 & 0.57 & $\beta$-agonist \\
\hline 8 & 26 & M & + & 86 & 100 & 0.74 & 0.08 & $\beta$-agonist \\
\hline 9 & 36 & $F$ & + & 67 & 73 & 0.77 & 0.97 & - \\
\hline 10 & 41 & F & + & 97 & 105 & 0.77 & 0.50 & - \\
\hline Mean & 32.5 & & & 72.9 & 85.9 & 0.71 & 0.29 & \\
\hline SD & 5.9 & & & 17.4 & 13.9 & 0.09 & 0.30 & \\
\hline \multicolumn{9}{|l|}{ Normals } \\
\hline 11 & 33 & $\mathbf{M}$ & - & 99 & 111 & 0.76 & $>75$ & - \\
\hline 12 & 33 & $\mathbf{M}$ & + & 109 & 114 & 0.81 & $>75$ & - \\
\hline 13 & 28 & $\mathbf{M}$ & + & 106 & 108 & 0.84 & $>75$ & - \\
\hline 14 & 26 & $F$ & - & 85 & 87 & 0.84 & $>75$ & - \\
\hline 15 & 47 & $F$ & - & 95 & 101 & 0.78 & $>75$ & - \\
\hline 16 & 31 & M & - & 125 & 131 & 0.82 & $>75$ & - \\
\hline 17 & 25 & $F$ & - & 94 & 99 & 0.83 & $>75$ & - \\
\hline 18 & 36 & $\mathbf{M}$ & - & 94 & 102 & 0.78 & $>75$ & - \\
\hline 19 & 49 & $\mathrm{~F}$ & - & 73 & 76 & 0.78 & $>75$ & - \\
\hline 20 & 32 & $F$ & + & 97 & 94 & 0.88 & $>75$ & - \\
\hline Mean & 34 & & & 97.7 & 102.3 & 0.81 & $>75$ & \\
\hline SD & 8.1 & & & 13.9 & 15.2 & 0.04 & 0 & \\
\hline
\end{tabular}

* $\mathrm{mg} / \mathrm{ml}$. prn, as needed.

\section{Subjects}

We studied 10 asthmatics and 10 nonasthmatics ranging in age from 24 to $49 \mathrm{yr}$ old (Table I). Asthmatic subjects fulfilled the diagnostic criteria of the American Thoracic Society (7) and were randomly selected from a large database of allergic/asthmatic individuals recruited from the community by radio/newspaper advertising. Nonasthmatic subjects were all employees of Johns Hopkins University. They reported no symptoms consistent with asthma and had never received the diagnosis of asthma from a physician. All subjects were screened by completing an asthma symptom questionnaire and undergoing allergy skin testing and routine Mch inhalation challenge. There was no significant difference in the mean age of the two groups. All 10 asthmatic subjects had multiple positive skin prick tests to common allergens and reacted to Mch with a provocative concentration $\left(\mathrm{PC}_{20}\right)<1 \mathrm{mg} / \mathrm{ml}$. The asthmatic subjects had significantly lower forced expiratory volume $\left(\mathrm{FEV}_{1}\right) \%$ predicted, forced vital capacity (FVC) $\%$ predicted, and $\mathrm{FEV}_{1} / \mathrm{FVC}$ compared to the normal subjects $(P<0.002, P<0.02$, and $P<0.01$, respectively). Three of the nonasthmatic subjects had positive skin prick tests but not related to any upper or lower respiratory symptoms. All of the normal subjects received the highest concentrations of Mch in the challenge $(75 \mathrm{mg} / \mathrm{ml})$ with no more than $15 \%$ reduction in $\mathrm{FEV}_{1}$. All subjects were nonsmokers, had been free of upper respiratory infection for at least $4 \mathrm{wk}$ before evaluation, and abstained from caffeine on Mch challenge testing days. Asthmatic subjects withheld all asthma medications before being studied ( $48 \mathrm{~h}$ for theophylline preparations and $8 \mathrm{~h}$ for inhaled $\beta$-agonists).

\section{Study design}

The study required a total of two visits to the laboratory. On one visit, all subjects underwent a screening evaluation as described above.

Routine methacholine challenge. As part of the screen, Mch challenge was conducted routinely; i.e., measurements of FEV ${ }_{1}$ and FVC were made $3 \mathrm{~min}$ after inhalation of a diluent (a combination of sodium chloride, sodium phosphate, potassium phosphate, and phenol) followed by bronchoprovocation with Mch. The initial concentration of Mch was $0.025 \mathrm{mg} / \mathrm{ml}$ and the concentration was increased in approximately half$\log$ increments $(0.025,0.075,0.25,0.75$, etc.) until a $20 \%$ decrease in $\mathrm{FEV}_{1}$ from diluent values was obtained or until the maximum concentration, $75 \mathrm{mg} / \mathrm{ml}$, was delivered. The provocative concentration of Mch causing this fall was calculated by interpolating the dose-response curve. Each concentration of the challenge solution was inhaled with five deep breaths from functional residual capacity to total lung capacity (TLC). The inhalation of Mch was made using a Rosenthal-French dosimeter (0.6-s actuation) and a DeVilbiss 646 nebulizer (DeVilbiss Co., Somerset, PA). At each dose level, a minimum of three reproducible forced expirations from TLC were performed, and the expiration with the highest $\mathrm{FEV}_{1}$ was used for analysis.

Modified methacholine challenge. On another visit, a modified Mch inhalation challenge was carried out. In the beginning, all subjects performed three reproducible consecutive maneuvers consisting of a forced expiration from end-tidal inspiration to residual volume (RV) (partial expiration) followed immediately by a deep inspiration to TLC and then, without breath holding, a forced expiration to RV (maximal expira- 
$\mathbf{A}$

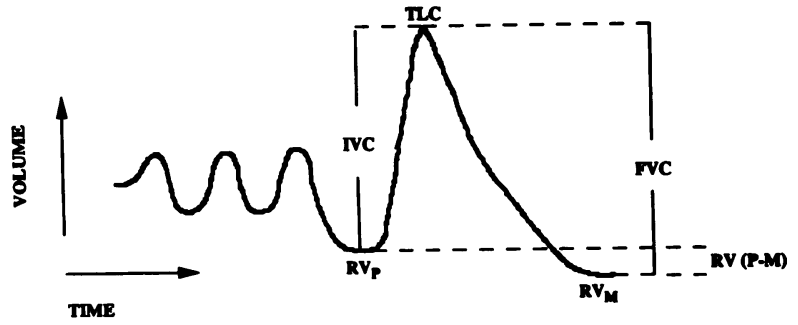

B

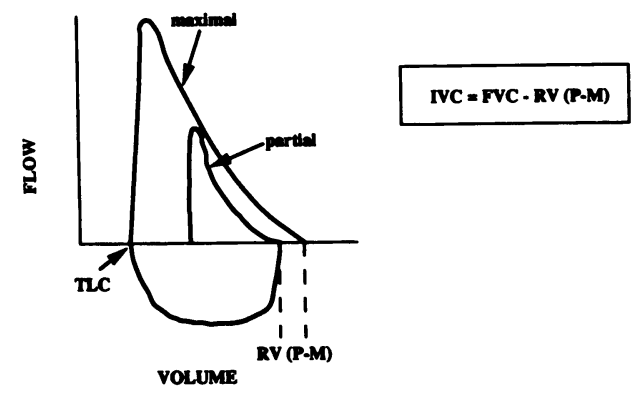

Figure 1. Example of a spirographic tracing $(A)$ and a flow-volume curve $(B)$ depicting the combination (partial followed by maximal) forced expiratory maneuver. After a series of three tidal breaths, subjects exhale forcefully from end-tidal volume to residual volume, then inhale to total lung capacity $(T L C)$ and immediately exhale forcefully to residual volume. $\left(R V_{P}\right)$ Residual volume after the partial expiratory maneuver; $\left(R V_{M}\right)$ Residual volume after the maximal expiratory maneuver; $[R V(P-M)]$ The difference in residual volumes between that obtained after the partial expiratory maneuver and that after the maximal expiratory maneuver.

tion) (Fig. 1). The partial and maximal forced expirations could be displayed as volume versus time (Fig. $1 \mathrm{~A}$ ) or flow versus volume (Fig. $1 B$ ). A Collins water-sealed spirometer (Warren E. Collins, Inc., Braintree, MA) with an attached potentiometer provided a voltage signal that was processed by a digital computer for the volume-time and flowvolume data. After these three partial and full maneuvers, here onto referred to as combination maneuvers, deep inspirations were prohibited during the entire time of the modified Mch challenge (We prohibited, but did not monitor, so we do not know whether occasional deep breaths or sighs occurred, but we saw no evidence that the participants were not following the instructions.). The modified challenge started with three partial forced expirations from end-tidal inspiration to RV. These were used as baseline for the subsequent steps of the Mch challenge. Each partial forced expiration to RV was followed by normal tidal breathing. Subjects then underwent Mch provocation with the diluen as the first dose. Each dose was administered as five tidal breaths from the dosimeter and nebulizer. 3 min later, three forced expirations from end-tidal inspiration were carried out as in the baseline partial forced maneuvers.

The modified challenge used the same starting concentrations and increments of Mch as the routine challenge. We monitored the change in pulmonary function by using the ratio of the volume expired during the partial forced expiration in $1 \mathrm{~s}$ divided by the total volume of the partial expiration (partial $\mathrm{FEV}_{1} / \mathrm{FVC}$ ). For each dose level, the partial maneuver with the highest $\mathrm{FEV}_{1}$ and that with the highest FVC were used to determine the partial $\mathrm{FEV}_{1} / \mathrm{FVC}$. We used the ratio of the volume expired in $1 \mathrm{~s}$ to the total volume expired during the partial maneuver rather than either absolute volume alone, because we were concerned that the absolute volumes were dependent on the end-tidal volume that was under the voluntary control of the subject. In both the normal and asthmatic subjects, we usually administered Mch until the partial $\mathrm{FEV}_{1} / \mathrm{FVC}$ fell to a value between 0.55 and 0.5 . If uncomfortable chest symptoms occurred before this level was reached, we gave no further inhalations of Mch. After the highest dose of Mch was reached, the subject repeated the three combination maneuvers that had been previously performed prior to the challenge.

Lung function analysis during the modified methacholine challenge: the $\tau$ index. While we monitored the change during the modified Mch challenge by the change in the partial $\mathrm{FEV}_{1} / \mathrm{FVC}$, we did not use this value when we analyzed the dose-response relations. Although this ratio decreases as airway constriction increases, the quantitative relation between the degree of bronchoconstriction and a specific change in the ratio is very complex. On the other hand, the time relations of a forced expiration are more suitable for quantitative analysis $(8,9)$. We chose to assess the time relations of the partial forced expirations from the reciprocal of the partial mid maximal expiratory flow (MMEF) FVC. (The time relations of the partial forced expiration could also have been made from the reciprocal of $\mathrm{FEV}_{1} / \mathrm{FVC}$. As bronchoconstriction increases, the portion of the forced expiration covered by the volume expired in one second progressively decreases, but the MMEF/FVC always covers the middle portion of the forced expiration.) This ratio was determined from the highest MMEF and the highest FVC from the three reproducible partial expiratory curves. The reciprocal of MMEF/ FVC is exactly equal to twice the time taken to expire the middle half of the forced expiration. If the reciprocal is multiplied by a specific constant $(0.5 / \ln 3)$, the resulting index is the reciprocal of the mean slope of the flow-volume curve between 25 and $75 \%$ of the forced expiration. This reciprocal slope is in units of time and can be considered the average time constant $(\tau)$ of the middle portion of the forced expiration (10). This index $(\tau)$ is directly related to the product of the average values of lung compliance and resistance to flow between the alveoli and the site of flow limitation in the airways $(10,11)$. Since the baseline values of $\tau$ were different in the asthmatic and normal subjects, we used the fractional change in $\tau$ from baseline (the ratio of the difference between $\tau$ at a specific dose of Mch and $\tau$ at baseline relative to the baseline $\tau$ ) as our primary outcome value (this is the percent change divided by 100 ).

Evaluation of changes in residual volume. We assumed that TLC remained constant during challenge (12). We also assumed that changes in the volume inspired from RV to TLC, the inspiratory vital capacity (IVC), are equal to the changes in RV that occurred during the period of challenge where deep breaths were prohibited and that, after deep inspiration maneuvers, the changes in FVC were equal to the changes in RV. The change in IVC was measured as follows: from each combination maneuver the difference in the RV between the partial and the maximal expiration $[R V(P-M)]$ was calculated from the flow-volume curves (Fig. $1 B$ ). The IVC was measured by subtracting RV $(P-M)$ from FVC (Fig. $1 A$ ). In the initial combination maneuvers, the average $\mathrm{RV}(\mathrm{P}-\mathbf{M})$ from the three reproducible curves was subtracted from the average FVC for the measurement of IVC. The IVC for each of the three combination maneuvers that was performed after the highest dose of Mch was calculated independently.

Evaluation of changes in isovolume flow. With the assumption of constant TLC, we linearized the flow-volume curve by a straight line from RV with a slope of $1 / \tau$. The decrease in isovolume flow is then a function both of the increase in $\tau$ and the increase in RV. The ratio of flow at the highest Mch dose to the flow at baseline at a forced expired volume one-half of the baseline FVC (from TLC) was the index used. This ratio was estimated both for the partial maneuver ( $\left.R \dot{v} .5_{\mathrm{P}}\right)$ and the first maximal breath $\left(R \dot{v} .5_{\mathrm{M}}\right)$ from the combination maneuvers.

Statistical analysis. Within and between group comparisons of baseline values, of the effect of Mch during the modified Mch challenge protocol, and of the effect of a deep breath following the modified Mch challenge protocol, were performed using two-tailed, paired or unpaired Student's $t$ test, respectively.

Two-way ANOVA with group (asthmatics versus normals) and breath (1st, 2nd, 3rd) main and interactive effects was used to compare differences between the three full breaths that followed the highest dose of Mch in the modified challenge. If significance was obtained with ANOVA, post hoc analysis using Dunnett's $t$ test was done.

Data are presented in the figures and tables as mean \pm SEM except 
Table II. Mean Pulmonary Function Values \pm SEM at Baseline and at the End of the Methacholine Challenge in the Modified Methacholine Provocation Protocol

\begin{tabular}{|c|c|c|c|c|c|c|c|}
\hline \multicolumn{4}{|c|}{ Maximal forced expiratory maneuvers } & \multicolumn{4}{|c|}{ Partial forced expiratory maneuvers } \\
\hline Variable & $\begin{array}{l}\text { Normals } \\
(n=10)\end{array}$ & $\begin{array}{l}\text { Asthmatics } \\
(n=10)\end{array}$ & $P$ value & Variable & $\begin{array}{l}\text { Normals } \\
(n=10)\end{array}$ & $\begin{array}{l}\text { Asthmatics } \\
(n=10)\end{array}$ & $P$ value \\
\hline \multicolumn{8}{|l|}{ Baseline } \\
\hline $\mathrm{FEV}_{1} / \mathrm{FVC}$ & $0.809 \pm 0.014$ & $0.708 \pm 0.023$ & $<0.002$ & $\mathrm{FEV}_{1} / \mathrm{FVC}$ & $0.743 \pm 0.02$ & $0.598 \pm 0.032$ & $<0.002$ \\
\hline$\tau \mathrm{B}_{\mathrm{M}}(\mathrm{s})$ & $0.574 \pm 0.034$ & $0.954 \pm 0.098$ & $<0.002$ & $\tau \mathrm{B}_{\mathrm{P}}(\mathrm{s})$ & $0.819 \pm 0.063$ & $1.491 \pm 0.162$ & $<0.002$ \\
\hline$\dot{\mathrm{V}} .5 \mathrm{~B}_{\mathrm{M}}$ (liter/s) & $4.044 \pm 0.384$ & $2.145 \pm 0.245$ & $<0.001$ & $\dot{\mathrm{V}} .5 \mathrm{~B}_{\mathrm{P}}($ liter$/ \mathrm{s})$ & $3.218 \pm 0.403$ & $1.485 \pm 0.186$ & $<0.002$ \\
\hline $\mathrm{FEV}_{1}$ (liter) & $3.773 \pm 0.386$ & $2.665 \pm 0.192$ & $<0.02$ & & & & \\
\hline FVC (liter) & $4.689 \pm 0.496$ & $3.754 \pm 0.029$ & NS & & & & \\
\hline IVC (liter) & $4.851 \pm 0.518$ & $3.868 \pm 0.199$ & NS & & & & \\
\hline \multicolumn{8}{|c|}{ After highest methacholine dose } \\
\hline $\mathrm{FEV}_{1} / \mathrm{FVC}$ & $0.682 \pm 0.02$ & $0.622 \pm 0.022$ & NS & $\mathrm{FEV}_{1} / \mathrm{FVC}$ & $0.528 \pm 0.021$ & $0.494 \pm 0.025$ & NS \\
\hline$\tau \mathrm{A}_{\mathrm{M}}(\mathrm{s})$ & $0.924 \pm 0.063$ & $1.314 \pm 0.097$ & $<0.002$ & $\tau \mathrm{A}_{\mathrm{P}}(\mathrm{s})$ & $1.903 \pm 0.131$ & $2.018 \pm 0.127$ & NS \\
\hline$\dot{\mathrm{V}} .5 \mathrm{~A}_{\mathrm{M}}(\mathrm{liter} / \mathrm{s})$ & $1.374 \pm 0.356$ & $0.823 \pm 0.133$ & NS & $\dot{\mathrm{V}} .5 \mathrm{~A}_{\mathrm{P}}($ liter $/ \mathrm{s})$ & $0.588 \pm 0.143$ & $0.541 \pm 0.067$ & NS \\
\hline $\mathrm{FEV}_{1}$ (liter) & $2.473 \pm 0.399$ & $1.822 \pm 0.161$ & NS & & & & \\
\hline FVC (liter) & $3.587 \pm 0.532$ & $2.937 \pm 0.234$ & NS & & & & \\
\hline IVC (liter) & $3.416 \pm 0.501$ & $2.986 \pm 0.213$ & NS & & & & \\
\hline
\end{tabular}

The left panel values (maximal maneuvers) derive from the segments of the protocol that preceded and followed the methacholine challenge. In these segments, full breaths were performed as part of the partial/maximal combination maneuvers which are described in the method section and in Fig. 1. The right panel values derive from the segment of the protocol during which increasing doses of methacholine were given and deep breaths were prohibited (partial maneuvers). $P$ values derive from the comparisons between normals and asthmatics. $\tau$ B $\mathrm{B}_{\mathrm{M}}: \tau$ from baseline maximal maneuver; $\tau \mathrm{A}_{\mathrm{M}}: \tau$ from first maximal maneuver after the highest dose of methacholine; $\tau \mathrm{B}_{\mathrm{p}}: \tau$ from baseline partial maneuver; $\tau$ Ap: $\tau$ from partial maneuver after the highest dose of methacholine; $\dot{V} .5 \mathrm{~B}_{\mathrm{M}}$ : flow on first maximal maneuver at baseline at a volume $50 \%$ of the maximal effort baseline FVC (from TLC); $\dot{V} .5 \mathrm{~A}_{\mathrm{M}}$ : flow on first maximal maneuver after the highest dose of methacholine at a volume $50 \%$ of the maximal effort baseline FVC (from TLC); $\dot{V} .5 B_{\mathrm{p}}$ : flow on partial maneuver at baseline at a volume $50 \%$ of the maximal effort baseline FVC (from TLC); $\dot{V} .5$ A : flow on partial maneuver after the highest dose of methacholine at a volume $50 \%$ of the maximal effort baseline FVC (from TLC).

for Table I where standard deviations were used. To analyze and depict the methacholine doses, geometric means were used.

\section{Results}

The mean baseline measurements and those following the highest concentration of Mch during the modified protocol are shown in Table II. Two types of data are indicated: those that were obtained during the period where deep inspiration (DI) was prohibited and those where measurements for both the baseline and the response to Mch were obtained from the combination maneuvers that required full breaths from RV to TLC (IVC) and/or from TLC to RV (FVC).

We attempted to give a dose of Mch that reduced the partial $\mathrm{FEV}_{1} / \mathrm{FVC}$ in all subjects to approximately the same level, and this we essentially achieved. The partial $\mathrm{FEV}_{1} / \mathrm{FVC}$ at the highest dose of Mch in the asthmatics was not significantly different from the value in the normal subjects, and $\tau$ reached a value in both groups that was virtually identical (Table II). To reach essentially the same endpoint required a significantly greater dose of Mch in the normal subjects than the asthmatics (geometric mean \pm SEM of the final concentration of Mch for normal subjects: $10.8 \pm 1.41 \mathrm{mg} / \mathrm{ml}$; for asthmatic subjects: $0.39 \pm 1.47 \mathrm{mg} / \mathrm{ml}, P<0.0001)$. The baseline partial $\mathrm{FEV}_{1} /$ FVC was significantly greater in the normal than the asthmatic subjects and the partial $\tau$, significantly less $(P<0.002)$. Thus, the fractional response in $\tau$ was considerably greater in the normal subjects $(P<0.005)$. Considering response a function of fractional change rather than end-point reached, the greater fractional change in the normal subjects is in keeping with the greater dose of Mch.

The response of the normal and asthmatic subjects during the prohibition of DI was compared by examining the fractional change in $\tau$ at the same dose level in both groups. Both groups could be compared up to the level of $0.25 \mathrm{mg} / \mathrm{ml}$. This level was reached by all of the normal subjects and by 9 of the 10

Table III. Mean Fractional Changes in the Index

\begin{tabular}{cccc}
\hline \multicolumn{4}{c}{ Methacholine concentration $(\mathrm{mg} / \mathrm{ml})$} \\
\hline Diluent & 0.025 & 0.075 & 0.25 \\
\hline Normals (N) & & & \\
$0.042 \pm 0.026$ & $0.038 \pm 0.056$ & $0.128 \pm 0.043$ & $0.245 \pm 0.065$ \\
$n=10, \mathrm{NS}$ & $n=10, \mathrm{NS}$ & $n=10, p<0.03$ & $n=10, P<0.01$ \\
Asthmatics (A) & & & \\
$0.040 \pm 0.048$ & $0.032 \pm 0.029$ & $0.113 \pm 0.041$ & $0.263 \pm 0.058$ \\
$n=10, \mathrm{NS}$ & $n=10, \mathrm{NS}$ & $n=9, P<0.07$ & $n=9, P<0.005$ \\
$\mathrm{~N}-\mathrm{A}$ & & & \\
$0.002 \pm 0.053$ & $0.006 \pm 0.065$ & $0.015 \pm 0.060$ & $-0.018 \pm 0.088$ \\
NS & NS & NS & NS \\
& & & \\
\hline
\end{tabular}

Mean fractional changes \pm SEM from baseline in the index $\tau$ after diluent and after the first three doses of methacholine, in the course of the modified methacholine provocation ( $\tau$ after each step minus $\tau$ at baseline divided by $\tau$ at baseline). The data derive from the partial maneuvers performed in the course of this protocol (deep breaths prohibited). Within group statistical comparisons are against $0 . \mathrm{N}$ - A: difference between normals and asthmatics. 


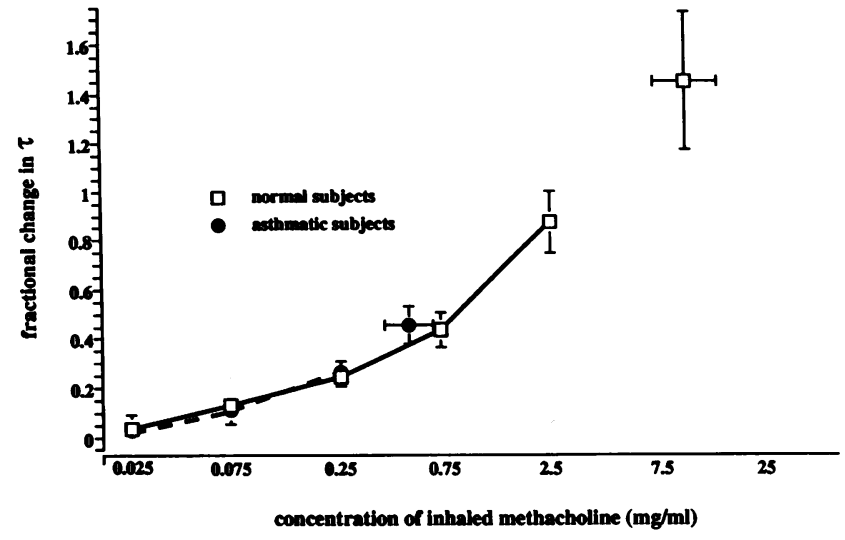

Figure 2. Dose response curves of the methacholine-induced fractional changes in the index $\tau$ from baseline ( $\tau$ after methacholine minus $\tau$ at baseline divided by $\tau$ at baseline) in the normal and in the asthmatic subjects, during the modified methacholine challenge protocol (deep breaths prohibited). Values represent means \pm SEM. In addition to the values at each concentration of methacholine, the figure depicts the average fractional change in $\tau$ at the geometric mean of the highest concentration of delivered methacholine for the normal and the asthmatic groups independently. The horizontal error bars of these values represent the SEM of the highest concentration of methacholine delivered to each subject group.

asthmatic subjects. The results are summarized in Table III and in Fig. 2.

Neither the asthmatics nor the normal subjects responded to the diluent or the $0.025 \mathrm{mg} / \mathrm{ml}$ dose. For the $0.075 \mathrm{mg} / \mathrm{m}$ dose, there was a significant fractional increase in $\tau$ in the normal subjects by the two-tailed $t$ test and a significant fractional increase in $\tau$ in the asthmatic subjects by the one-tailed $t$ test, but there was no difference between the groups. At 0.25 $\mathrm{mg} / \mathrm{ml}$, there was a significant fractional increase in both groups by the two-tailed test, and again there was no difference between the two groups. Also shown in Fig. 2 is the mean fractional change in $\tau$ at the geometric mean level of the highest dose reached.

The effectiveness of DI in decreasing the response produced by the highest level of Mch was made by comparing the change from baseline in either $\tau$ or RV that occurred during the period of prohibition of DI to the change from baseline measured after the first full breath that followed the prohibition of DI. From the changes in $\tau$ and $R V, R \dot{v} .5_{\mathrm{P}}$ and $\mathrm{R} \dot{v} .5_{\mathrm{M}}$ were also calculated.

The fractional change from baseline in $\tau$ was significantly reduced by DI only in the normal subjects (Fig. 3). Similarly, the change in RV was significantly reduced by DI only in the normal subjects (Fig. $4 A$ ). However, in the normal subjects, $\tau$ was still significantly elevated over baseline and a large increase in RV (average of $1.1 \pm 0.18$ liter) remained even after DI. When the change in RV was considered as a fractional change of the baseline FVC, again there was a significant effect of DI in reducing the change only in the normal subjects (Fig. $4 B$ ). Finally, if the difference in the change in RV produced by $\mathrm{DI}\left(\Delta \mathrm{RV}_{\mathrm{P}}-\Delta R \mathrm{~V}_{\mathrm{M}}\right)$ was considered as a fraction of the change in $R V$ produced in the absence of $D I\left(\Delta R V_{P}\right)$, the effect of DI on this ratio $\left[\left(\Delta R V_{P}-\Delta R V_{M}\right) / \Delta R V_{P}\right]$ was significantly $>0$ only in the normal subjects (Fig. $4 C$ ).

The effect of DI was an increase in Rvं.5 (a reversal of the Mch-induced decrease in isovolume flow) in the normal
NORMAL SUBJECTS

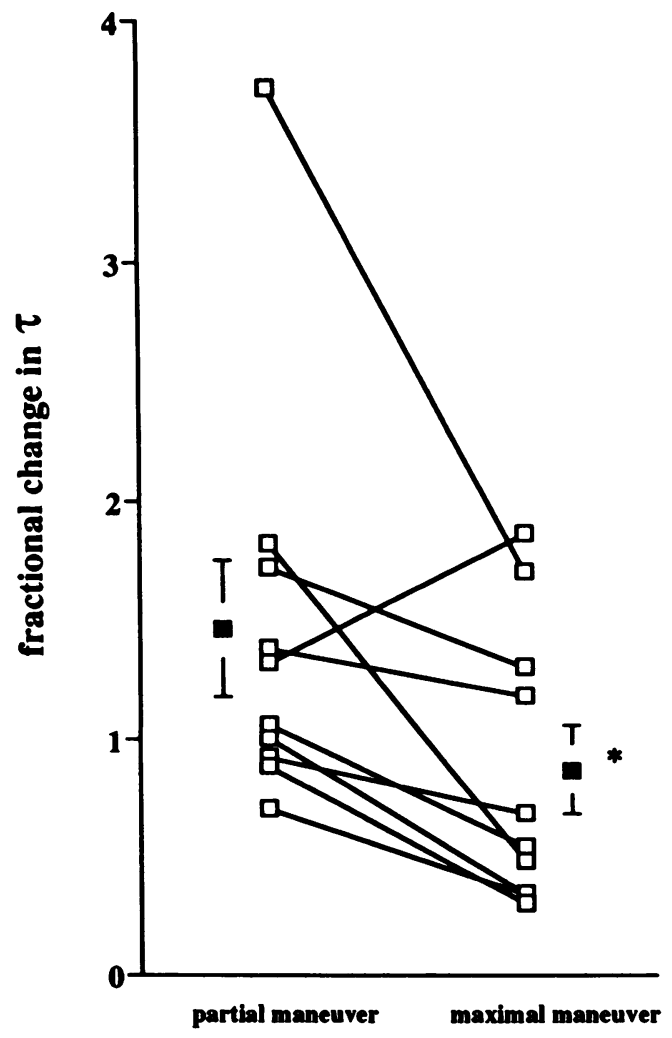

\section{ASTHMATIC SUBJECTS}

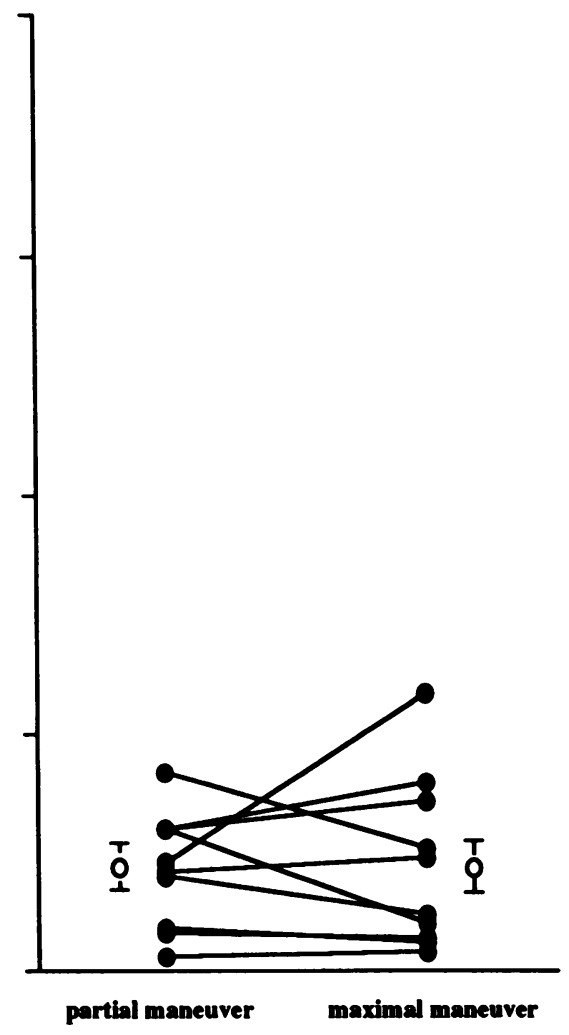

Figure 3. Modified methacholine challenge protocol: comparison between partial and maximal expiratory maneuvers performed after the highest dose of methacholine. Individual data and means \pm SEM for the norma and the asthmatic subjects are depicted. The outcome variable is the methacholine-induced fractional change in the index $\tau$ from baseline ( $\tau$ after methacholine minus $\tau$ at baseline divided by $\tau$ at baseline). The left values on each panel are the differences between $\tau_{\mathrm{A}_{\mathrm{p}}}$ and $\tau_{\mathrm{B}_{\mathrm{p}}}$ divided by $\tau_{B_{\mathrm{p}}}$. The right values are the differences between $\tau_{\mathrm{A}_{M}}$ and $\tau_{\mathrm{B}_{\mathrm{M}}}$ divided by $\tau_{\mathrm{B}_{\mathrm{M}}}$ ( see the legend of Table II for explanation of these terms ). $* P<0.05$ between the maximal and the partial maneuvers in the normal subjects. 
$\mathbf{A}$
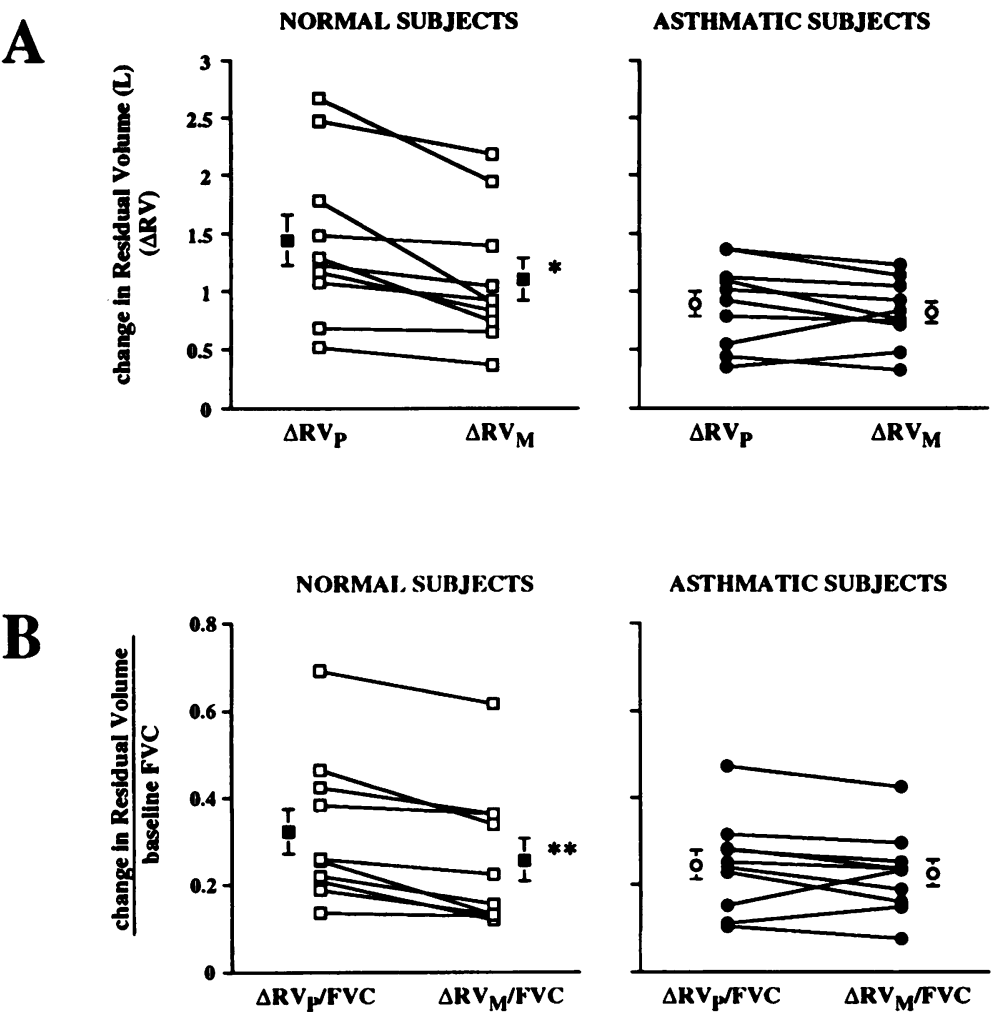

C

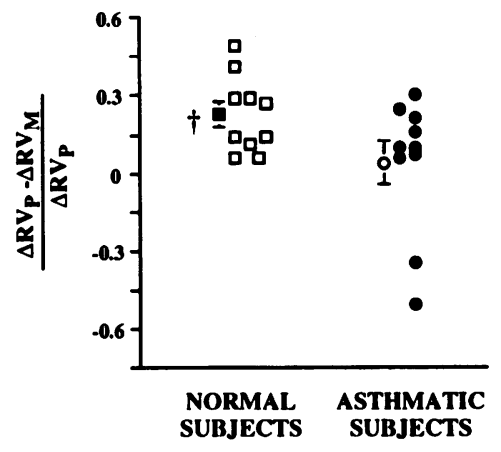

ASTHMATIC SUBJECTS

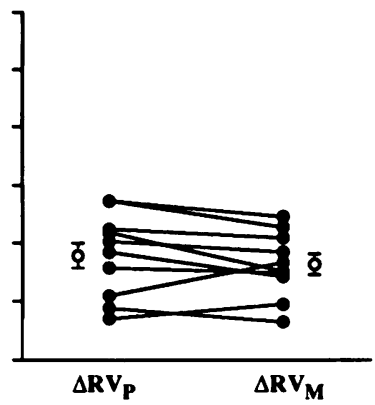

Figure 4. Modified methacholine challenge protocol: the effect of deep inspiration on the change in residual volume at the highest dose of methacholine in relation to baseline residual volume. Individual data and means \pm SEM for the normal and the asthmatic subjects are depicted. $\Delta \mathrm{RV}$ : methacholine-induced change in residual volume from baseline; $(A)$ The values on the left of each panel $\left(\Delta R V_{P}\right)$ are from the partial maneuvers $\left(R V_{\mathrm{A}_{\mathrm{p}}}-R V_{\mathrm{B}_{\mathrm{p}}}\right)$; the values on the right $\left(\triangle R V_{M}\right)$ are from the maximal maneuvers $\left(\mathrm{RV}_{\mathrm{A}_{\mathrm{M}}}-\mathrm{RV}_{\mathrm{B}_{\mathrm{M}}}\right){ }^{*} P<0.005$ between the maximal and the partial effort in the normal subjects. $R V_{B_{M}}$ : residual volume from baseline maximal maneuver; $\mathbf{R V}_{\mathrm{B}_{\mathrm{p}}}$ : residual volume from baseline partial maneuver; $\mathrm{RV}_{\mathrm{A}_{\mathrm{M}}}$ : residual volume from maximal maneuver after the highest dose of methacholine; $\mathrm{RV}_{\mathrm{AP}}$ : residual volume from partial maneuver after the highest dose of methacholine. (B) The outcome variable is the methacholine-induced change from baseline in residual volume $(\Delta R V)$ divided by baseline FVC. The values on the left of each panel are from the partial maneuvers; the values on the right are from the maximal maneuvers. ${ }^{* *} P<0.001$ between the maximal and the partial effort values in the normal subjects. $(C)$ The outcome variable represents the deep breath-induced change in residual volume relative to the increase in residual volume produced by methacholine challenge in the absence of deep breaths. $\Delta \mathrm{RV}_{\mathrm{P}}$ and $\Delta \mathrm{RV}_{\mathrm{M}}$ are the same as in $A .{ }^{\dagger} P<0.001$ between normal and asthmatic subjects. subjects, but this effect was absent in the asthmatic subjects (Fig. 5).

The change in $\mathrm{FEV}_{1}$ and FVC with Mch was much greater in the modified than the routine challenge in the normal subjects, but this was not so for the asthmatic subjects (Fig. 6, $A$ and $B$ ). The increased response in the modified challenge in the normal subjects was associated with a smaller dose of Mch. Indeed, in the modified challenge, 6 of the 10 normal subjects reached the challenge endpoint at a dose conventionally considered within the asthmatic range $(<8 \mathrm{mg} / \mathrm{ml})$. In contrast, in the asthmatic group, the same number of doses of Mch were used to reach the endpoint in both the routine and the modified challenge (Fig. $6 \mathrm{C}$ ). In the modified challenge, the fractional change from baseline in both $\mathrm{FEV}_{1}$ and FVC became less with subsequent breaths in both the normal and the asthmatic subjects, but the effect of the subsequent breaths in increasing $\mathrm{FEV}_{1}$ and FVC was not significantly different between the normal and the asthmatic subjects (Table IV). However, there was borderline statistical significant $(P=0.11)$ for the group/breath interaction in this analysis. The difference between the modified and routine challenge in the normal subjects was still significant if the third (instead of the first) full breath that followed the modified challenge was used for both $\operatorname{FEV}_{1}(P<0.05)$ and FVC $(P<0.02)$ (data not shown).

Two additional studies were carried out on a subset of normal and asthmatic subjects to rule out any direct effect of the prohibition of DI. We repeated the modified challenge substituting diluent for Mch, and found that prohibition of DI in itself did not cause a significant decrease in pulmonary function (partial $\left.\mathrm{FEV}_{1} / \mathrm{FVC}\right)$ in either the nonasthmatic subjects $(n=5)$ or asthmatics $(n=4)$. The partial $\mathrm{FEV}_{1} / \mathrm{FVC}$ after the first dose of diluent compared to the last dose of diluent (five doses given to the nonasthmatics and four to the asthmatics) was $0.74 \pm 0.04$ versus $0.76 \pm 0.04$ in the nonasthmatics and $0.67 \pm 0.01$ versus $0.68 \pm 0.02$ in the asthmatics.

In five normal subjects and six asthmatic subjects, we car- 
NORMAL SUBJECTS

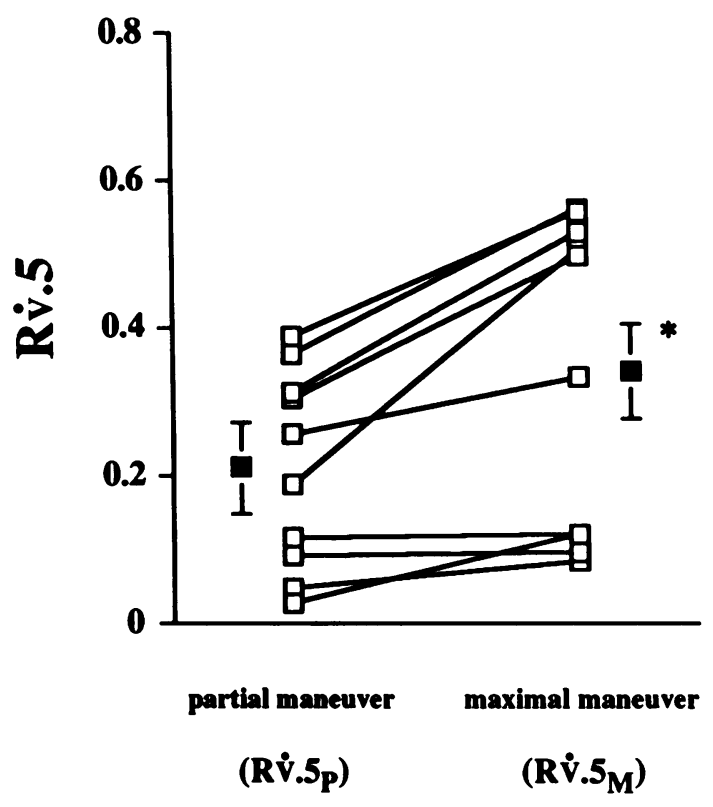

ASTHMATIC SUBJECTS

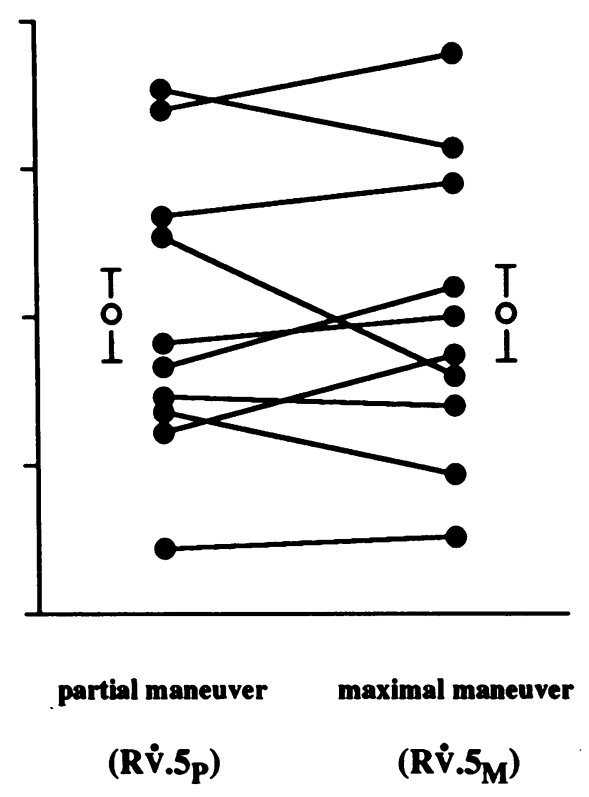

Figure 5. Modified methacholine challenge protocol: Comparison between partial and maximal expiratory maneuvers performed after the highest dose of methacholine. Individual data and means \pm SEM for the normal and the asthmatic subjects are depicted. The outcome variable $\left(R_{i}\right)$ is the ratio of the flow at the highest dose of methacholine to the flow at baseline (both flows are measured at a volume $50 \%$ below TLC of the maximal effort baseline FVC). $R_{i} \cdot 5_{p}$, the ratio of the flow on the partial curve at the highest dose of methacholine to the flow on the partial curve atbaseline; $R_{i} \cdot 5_{\mathrm{M}}$, the ratio of the flow on the maximal curve at the highest dose of methacholine to the flow on the maximal curve at baseline. ${ }^{*} P<0.005$ between the maximal and the partial effort values in the normal subjects. ried out routine Mch challenges with the inhalation of the Mch by tidal breaths rather than by deep inhalations as in our conventional Mch challenge. This study was performed to assess whether, by altering the pattern of inhalation of Mch, as in our modified challenge protocol, alterations in airway responsiveness could occur. In the normal subjects, we saw no evidence of increased responsiveness to $\mathrm{Mch}$, when it was inhaled through tidal breathing. For the asthmatic subjects, where $\mathbf{P C}_{20}$ could be obtained, there was a trend toward decreased reactivity when Mch was inhaled by tidal inhalations compared to the conventional deep ones $\left(\mathrm{PC}_{20}=0.29 \pm 0.14\right.$ versus $0.15 \pm 0.21$, $P=0.055)$.

\section{Discussion}

The hypothesis that led to this study, that hyperresponsiveness in asthma is caused by impaired ability of DI to dilate the airways, is fully supported by our primary findings: $(a)$ in the absence of DI, the airway response to Mch is similar in asthmatics and nonasthmatics, and $(b)$ in nonasthmatics, DI has a striking effect in preventing Mch-induced bronchoconstriction, but DI has no influence on the response to Mch-induced bronchoconstriction in asthmatic subjects.

All of the normal subjects and 9 out of 10 of the asthmatic subjects inhaled the same concentrations of Mch up to the level of $0.25 \mathrm{mg} / \mathrm{ml}$. Over this range, the fractional change in $\tau$ was virtually identical in both groups with a threshold of $0.075 \mathrm{mg}$ / $\mathrm{ml}$. The design of the study was such that we did not determine the change in RV until higher concentrations were reached in both groups of subjects. It is possible that the change in RV was greater in the asthmatic than the normal subjects at the same dose of Mch in spite of the same change in $\tau$. If this were so, and since the change in isovolume flow is a function of the change in RV and the change in $\tau$, there would have been a greater decrease in isovolume flow in the asthmatic subjects compared to the normal subjects.
The isovolume flows of the linearized flow-volume curves are given by the following equations:

$$
\begin{aligned}
& R \dot{v} .5_{P}=\left[1-\frac{2 \Delta R V_{P}}{F_{V C}-2 R V(P-M)}\right]\left[\frac{\tau_{B_{P}}}{\tau_{A_{P}}}\right] \\
& R \dot{v} .5_{M}=\left[1-\frac{2 \Delta R V_{M}}{F_{V C}}\right]\left[\frac{\tau_{B_{M}}}{\tau_{A_{M}}}\right]
\end{aligned}
$$

where: $R \dot{v} .5_{\mathrm{P}}=$ ratio of the flow on the partial curve at the highest dose of Mch to the flow on the partial curve at baseline, at a volume below TLC of $50 \%$ of the maximal baseline FVC. Riv. $5_{M}=$ ratio of the flow on the maximal curve at the highest dose of Mch to the flow on the maximal curve at baseline, at a volume below TLC of $50 \%$ of the maximal baseline FVC. $\Delta \mathrm{RV}_{\mathrm{P}}=$ change from baseline in $\mathrm{RV}$ during partial maneuvers ( $=-\Delta \mathrm{IVC}) . \Delta \mathrm{RV}_{\mathrm{M}}=$ change from baseline in $\mathrm{RV}$ during maximal maneuvers $(=-\Delta F V C) \cdot R V(P-M)=$ difference in RV between the partial and the maximal expirations from combination maneuvers prior to the Mch challenge (Fig. $1 B$ ). $\tau_{\mathrm{B}_{\mathrm{P}}}=\tau$ from baseline partial curve. $\tau_{\mathrm{B}_{\mathrm{M}}}=\tau$ from baseline maximal curve. $\tau_{\mathrm{Ap}_{\mathrm{p}}}=\tau$ from partial curve after highest dose of Mch inhaled. $\tau_{\mathrm{A}_{\mathrm{M}}}=\tau$ from first maximal curve after highest dose of Mch inhaled. $\mathrm{FVC}_{\mathrm{B}}=\mathrm{FVC}$ from the baseline maximal maneuver. Thus, the ratio of isovolume flows is the product of two factors such that:

$$
\begin{aligned}
& R \dot{v} .5_{P}=\left(\beta_{R_{\mathrm{P}}}\right)\left(\beta_{\tau_{\mathrm{P}}}\right) \\
& \text { Riv. } 5_{M}=\left(\beta_{R_{M}}\right)\left(\beta_{\tau_{M}}\right)
\end{aligned}
$$

where:

$$
\begin{aligned}
& \beta_{\mathrm{RV}_{\mathrm{P}}}=1-\frac{2 \Delta \mathrm{RV}_{\mathrm{P}}}{\mathrm{FVC}_{\mathrm{B}}-2 \mathrm{RV}(\mathrm{P}-\mathrm{M})} \quad \beta \tau_{\mathrm{P}}=\frac{\tau_{\mathrm{B}_{\mathrm{P}}}}{\tau_{\mathrm{A}_{\mathrm{P}}}} \\
& \beta_{\mathrm{RV}_{\mathrm{M}}}=1-\frac{2 \Delta \mathrm{RV}_{\mathrm{M}}}{\mathrm{FVC}_{\mathrm{B}}} \beta \tau_{\mathrm{M}}=\frac{\tau_{\mathrm{B}_{\mathrm{M}}}}{\tau_{\mathrm{A}_{\mathrm{M}}}}
\end{aligned}
$$


$\mathbf{A}$

B
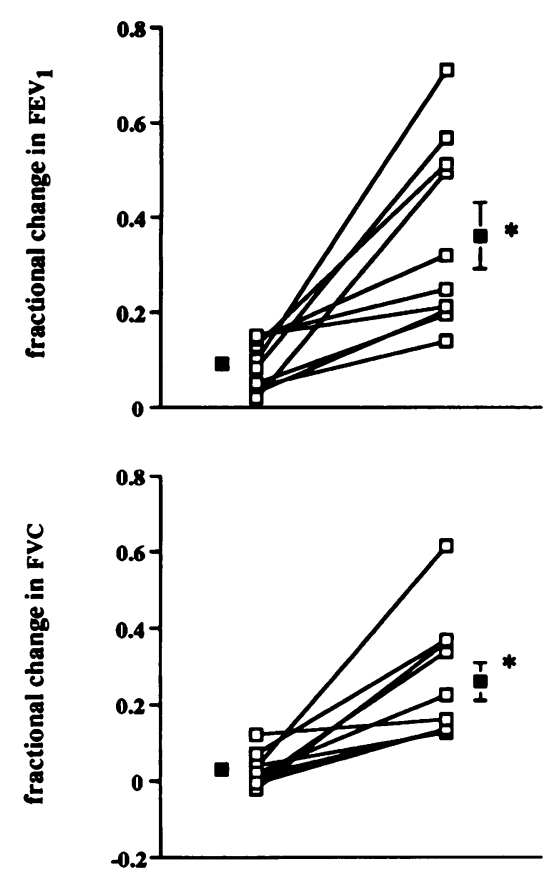

C

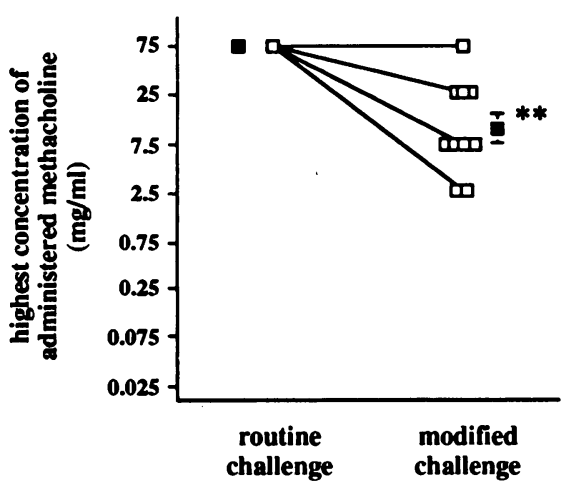

ASTHMATIC SUBJECTS
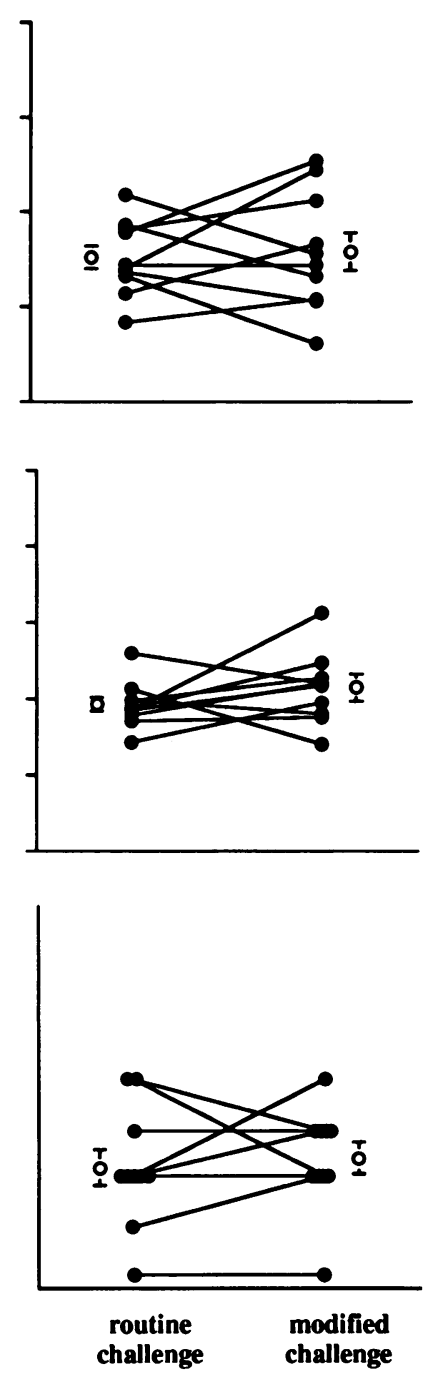

Figure 6. Individual data and means \pm SEM of the methacholine-induced fractional changes in $\mathrm{FEV}_{1}$ and in FVC ( $A$ and $B$, respectively) over baseline after the highest provocative dose in the routine and in the modified methacholine challenge protocols, in normal and in asthmatic subjects (fractional change $=\mathrm{FEV}_{1}$ or $\mathrm{FVC}$ after methacholine minus FEV 1 or FVC at baseline divided by FEV, or FVC at baseline). (C) Depicts the highest concentration of methacholine administered to each subject in each of the two protocols. $* P<0.01$ between routine and modified challenge; ${ }^{* *} P<0.001$ between routine and modified challenge.
Table IV. The Effect of Consecutive Deep Breaths on Pulmonary Function after the Highest Dose of Methacholine in the Modified Methacholine Challenge Protocol

\begin{tabular}{|c|c|c|c|c|}
\hline \multirow{2}{*}{$\begin{array}{l}\text { Post-Mch deep } \\
\text { breaths (from } \\
\text { combination } \\
\text { maneuvers) }\end{array}$} & \multicolumn{2}{|c|}{ Normals } & \multicolumn{2}{|c|}{ Asthmatics } \\
\hline & $\begin{array}{l}\text { Fractional } \Delta \\
\text { in FEV }\end{array}$ & $\begin{array}{l}\text { Fractional } \Delta \\
\text { in FVC }\end{array}$ & $\begin{array}{l}\text { Fractional } \Delta \\
\text { in } F^{\prime} V_{1}\end{array}$ & $\begin{array}{l}\text { Fractional } \Delta \\
\quad \text { in FVC }\end{array}$ \\
\hline First & $0.359 \pm 0.062$ & $0.258 \pm 0.051$ & $0.315 \pm 0.039$ & $0.225 \pm 0.030$ \\
\hline Second & $0.269 \pm 0.059$ & $0.195 \pm 0.056$ & $0.264 \pm 0.036$ & $0.185 \pm 0.027$ \\
\hline Third & $0.220 \pm 0.051$ & $0.146 \pm 0.042$ & $0.246 \pm 0.034$ & $0.170 \pm 0.025$ \\
\hline \multicolumn{5}{|l|}{ ANOVA table } \\
\hline \multicolumn{2}{|c|}{ For the fractional $\Delta$ in $\mathrm{FEV}_{1}$} & \multicolumn{3}{|c|}{$\begin{array}{l}\text { group effect: } P=0.90 \\
\text { breath effect: } P=0.0001 \\
\text { group/breath interaction: } P=0.11 \\
\text { group effect: } P=0.89 \\
\text { breath effect: } P=0.0001 \\
\text { group/breath interaction: } P=0.22\end{array}$} \\
\hline
\end{tabular}

The data (mean \pm SEM) are obtained from the maximal breaths which were part of combination maneuvers (partial followed by maximal). The fractional change $(\Delta)$ in $\mathrm{FEV}_{1}$ and in FVC equals the value at baseline minus the value after the respective post methacholine deep breath divided by the value at baseline.
The smaller each factor, the greater the effect on isovolume flow. In the asthmatic subjects, $\beta_{\mathrm{RV}}$ was significantly less than $\beta \tau$, both on the partial and the full maneuvers $(P<0.02$ and $P<0.05$, respectively, Table V). Thus, in the asthmatic subjects, the reduction in isovolume flow was more attributable to changes in RV than $\tau$. In the normal subjects, there was no difference between $\beta_{\mathrm{RV}}$ and $\beta_{\tau}$ either on the partial or maximal maneuvers (Table V). Thus, the reduction in isovolume flow in this group was essentially the result of equal effects of the changes in RV and $\tau$.

Since the fractional change in the partial $\tau$ was nearly identical in the normal and asthmatic subjects up to the highest dose of Mch achieved by the asthmatic subjects, we can assume that $\beta_{\tau_{\mathrm{p}}}$ was also the same ( $\beta_{\tau}$ is the reciprocal of $1+$ the fractional change in $\tau$ ). Since there was no difference between $\beta_{\tau_{\mathrm{p}}}$ and $\beta_{\mathrm{RV}_{\mathrm{P}}}$ in the normal subjects, we can assume that the reduction in isovolume flow at a Mch dose comparable to the highest dose reached in the asthmatic subjects was due to equal effects of the changes in $\mathrm{RV}$ and $\tau\left(\beta_{\tau_{\mathrm{P}}}=\beta_{\mathrm{RV}_{\mathrm{P}}}\right)$. Thus, we can estimate that, in the normals, the $R_{\mathrm{v}} \cdot 5_{\mathrm{P}}$, at the highest dose of Mch reached by the asthmatics, was the square of the asthmatics' $\beta_{\tau_{\mathrm{P}}}$ at their highest $\mathrm{Mch}$ dose $\left(\mathrm{R}_{\mathrm{v}} .5_{\mathrm{P}}\right.$ in normals $=0.723^{2}$ $=0.523)$. In contrast, in the asthmatics, the $R_{\mathrm{v}} .5_{\mathrm{P}}$ at the highest 
Table V. Isovolume Flow Ratios and their Residual Volume and Time Constant Determinants

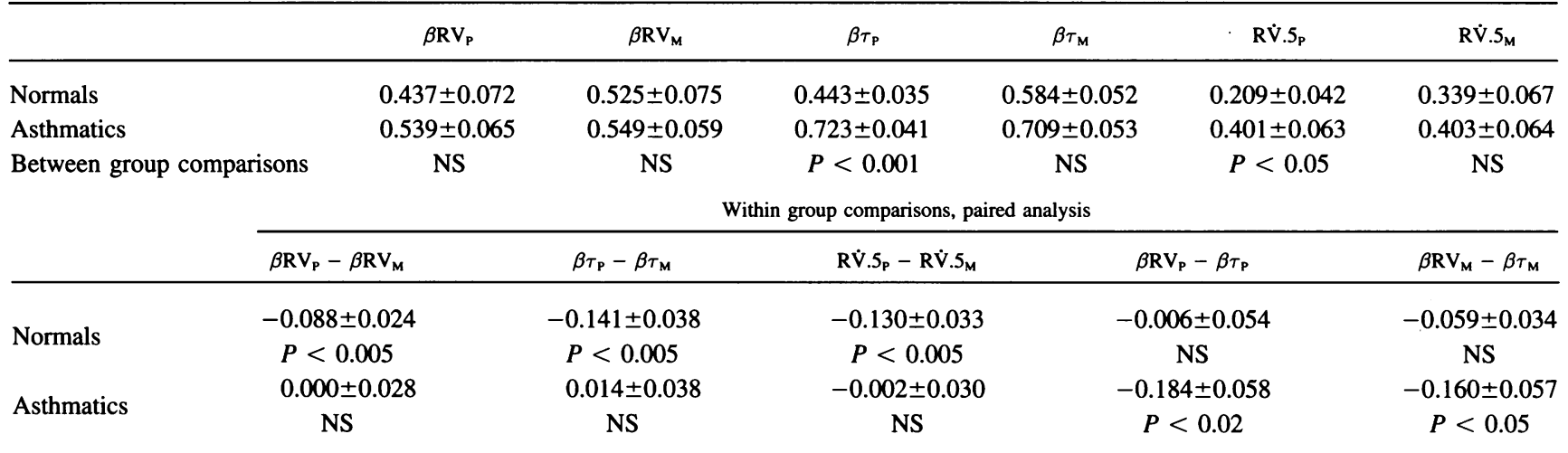

Between group and within group comparisons of the isovolume flow ratios RV $.5_{\mathrm{P}}$ and $\mathrm{R} \dot{\mathrm{V}} .5_{\mathrm{M}}$ (highest dose of methacholine to baseline) and their residual volume $\left(\beta \mathrm{RV}, \beta \mathrm{RV} \mathrm{V}_{\mathrm{M}}\right)$ and time constant $\left(\beta \tau_{\mathrm{P}}, \beta \tau_{\mathrm{M}}\right)$ determinants in the modified methacholine challenge protocol. $\mathrm{RV} \cdot \mathrm{J}_{\mathrm{P}}=\left(\beta \mathrm{RV} \mathrm{V}_{\mathrm{P}}\right)\left(\beta \tau_{\mathrm{P}}\right)$ and $R \dot{R} .5_{M}=\left(\beta R V_{M}\right)\left(\beta \tau_{M}\right)$. For definition of these terms refer to the Discussion section of the text. For the within group comparisons, the three left columns compare the differences between the maximal and partial maneuvers; the two right columns compare the relative effect of the changes in the residual volume to the changes in $\tau$ on the isovolume flow. Mean \pm SEM values are depicted.

Mch dose, was 0.401 (Table V). This lower value was the result of the significantly lower $\beta_{\mathrm{RV}_{\mathrm{P}}}$ in the asthmatic subjects. We estimate that in the asthmatic subjects, $R_{\mathrm{v}} .5_{\mathrm{p}}$ was $\sim 75$ $80 \%$ of the value of the normal subjects, and the estimated isovolume flow from the partial maneuvers, $25-30 \%$ less in the asthmatic than the normal subjects at the same dose.

Based on the statistically significant difference between $\beta_{\tau_{\mathrm{P}}}$ and $\beta_{\mathrm{RV}}$ in the asthmatic subjects but not the normal subjects, we infer that the response to inhaled Mch in the absence of DI was somewhat greater in the asthmatic subjects. However, the responses of the two groups were still close enough to lend support to the general correctness of the corollary of the Fish et al. hypothesis.

Despite the high concentrations of Mch they received (75 $\mathrm{mg} / \mathrm{ml}$ ), normal individuals experienced minimal bronchoconstriction with conventional Mch challenge. We believe that this was due to the stretching effect of DI which reversed the Mchinduced smooth muscle contraction. Based on this assumption, we expected that the modified Mch challenge-induced bronchoconstriction in nonasthmatic subjects should have been reversed with the first DI after the end of Mch administration. Surprisingly, even after the third DI of the post-Mch combination maneuvers, a large obstructive response was still present in nonasthmatics. This was true for all pulmonary function parameters evaluated. With the second and third DI, statistically significant improvement in pulmonary function was seen, but this was not different between normals and asthmatics (Table IV). In other words, not only does bronchoconstriction take place when the spasmogen is delivered in the absence of DI, but the ability of a healthy person to reverse the bronchoconstriction with a DI is strikingly reduced. This serendipitous observation is further substantiated when we compare our findings to those of two other studies in which the effects of DI on Mch-induced changes in RV were examined in normal and asthmatic subjects by the same method we used, that is, differences in IVC and FVC with the assumption of constant TLC (we are unaware of any studies other than ours where the time relations of a forced expiration were used to study the effects of DI).

In a recent study of Pellegrino et al. (13), the effect of DI on the fractional change in RV relative to baseline FVC was significantly greater in normal than in asthmatic subjects, qualitatively similar to the results of our study. In the normal subjects of their study, however, there was a much greater decrease in $\mathrm{RV}$ after DI than was found in our current study $(P<0.001$ by an unpaired $t$ test). Further, after DI in the normal subjects, the fractional increase in RV at the highest level of Mch was only 0.09 in the study of Pellegrino et al. in comparison to a fractional change of 0.26 in our current study. This difference was highly significant $(P<0.005)$ in spite of a significantly lower dose of Mch in our modified challenge in comparison to Pellegrino's study.

In a study of Wheatley et al. (14), the decrease in RV produced by DI relative to the change in RV before DI (the same index as in our Fig. $4 C$ ) was much greater in their normal subjects than we found in ours. (They used a slightly different index, but the index in Fig. $4 C$ can be calculated for each of the subjects they studied.) In their study, the ratio was 0.80 and in our study only $0.23(P<0.00005)$. Thus, the reversal by DI of the increase in RV in their normal subjects was nearly complete ( $80 \%$ reversal) in contrast to $<25 \%$ reversal in ours. As in our study, they found no significant reversal in asthmatic subjects.

In the studies of Pellegrino et al. and Wheatley et al., the response to Mch using partial and maximal expirations were carried out exactly the same as we did except there was no period of prohibition of deep inspirations. In their studies, a partial forced expiration to RV was always followed by a rapid inspiration to TLC with increasing concentrations of inhaled Mch in contrast to our study where the increasing concentrations of Mch were administered with prohibition of DI and only partial expirations were performed. We believe that the much greater effect of DI in reversing the increase in RV in the studies of Wheatley et al. and Pellegrino et al. in normal subjects in comparison to ours is likely due to the fact that our protocol involved repetitive partial forced expirations to RV with no intervening DI. If this inference is correct, the effectiveness of DI to decrease bronchoconstriction diminishes the greater the time spent in the constricted state at or near RV before the DI occurs. Further, if this inference is correct, we have an explanation to account for the marked difference in the magnitude of 
the decrease in FEV 1 and FVC in the modified versus the routine challenge in the normal subjects in our study (Fig. 6) and the rather slow improvement with the second and third full breaths (Table IV).

In the absence of DI during the inhalation of increasing concentrations of Mch, we believe that the repetitive partial forced expirations to RV are a necessity to produce marked responses in normal subjects. The mere absence of intervening DI is unlikely to be sufficient, because the marked responses of our normal subjects have not been observed in the numerous studies that have carried out dose-response relations using pulmonary resistance. Often, no information is given as to whether or not deep inspirations have been suppressed, but in a study of Ding et al. (15), the response of pulmonary resistance was measured under conditions where lung volume was controlled, and DI prohibited. In striking contrast to our results, the study of Ding et al. clearly demonstrated that small changes in lung elastic recoil pressure in the order of $2-3 \mathrm{~cm} \mathrm{H}_{2} \mathrm{O}$ markedly affected the response to Mch; whereas in our study, marked bronchoconstriction remained in the normal subjects even after full lung inflation. Further, our normal and asthmatic subjects had the same threshold concentration, but in the study of Ding et al. most of their normal subjects had high thresholds.

Airways are capable of complete closure at very high transmural pressures in vitro $(16,17)$. Gunst et al. found that maximal and even submaximal doses of Mch could produce airway closure in bronchi at constant transmural pressures in excess of $25 \mathrm{~cm} \mathrm{H}_{2} \mathrm{O}$ (18). The closure was completely prevented and the response to Mch markedly reduced if the bronchi were subjected to volume oscillations during the exposure to Mch. Thus, the in vitro work suggests that while volume oscillations during exposure to Mch can prevent closure, once the closure occurs, large forces must be generated to overcome the closure. These in vitro observations may provide an explanation for the results of the current study if DI in the normal subjects markedly attenuates the effect of inhaled Mch to cause closure; however, this effect is absent in patients with asthma. If the normal subjects do not invoke this method of attenuation, as in the modified challenge, the forces of closure become very great and persist even after maximal inspirations.

If the bronchodilating mechanism of DI is from stretch of the smooth muscle leading to stress relaxation, in keeping with the in vitro studies of Gunst et al. $(18,19)$, the attenuation of this mechanism in asthmatic subjects could be due to an increased stiffness of the airway wall, as suggested by the studies of Colebatch et al. (5). The asthmatic airway has increased thickness of the smooth muscle layer, increased collagen deposits beneath the basement membrane, and variable amounts of inflammatory products in the wall (20). It is also possible that the force dilating the airway is diminished in asthma. The mechanism by which intrapulmonary airways and smooth muscle within their walls are stretched by inspiration is largely due to the increase in radial traction on the airways exerted by the surrounding lung parenchyma (21-23). Edema of the airway wall or in the peribronchial space could unlink the interdependence between the airway and parenchyma resulting in a decrease in radial force acting on the smooth muscle with DI (24, $25)$. Ingram considered the interaction between the airways and lung parenchyma and proposed that the attenuated response to DI in asthma is due to a problem of increased parenchymal relative to airway hysteresis $(26,27)$.

Some consideration must be given to the possibility that the ineffectiveness of DI in asthmatic subjects is not due to a decrease in the stretch of smooth muscle. Even if DI produced the same degree of stretch in both groups, it is possible that the problem of asthma is a lack of responsiveness of the smooth muscle to stretch, either an intrinsic problem of asthmatic smooth muscle or a result of humoral inflammatory factors. Indeed, it has been suggested that asthmatic smooth muscle might show a myogenic response with an increase in tone accompanying stretch as in vascular smooth muscle, but this myogenic response is absent in nonasthmatic airways $(28,29)$. It is also possible that DI produces bronchodilatation through neural or humoral elements in normal subjects that are absent in asthmatic subjects. In a recent study of Malmberg et al. (30), a DI before the administration of Mch, in normal subjects, protected their airways from bronchoconstriction. This effect was even more pronounced than the effect of a DI after the administration of Mch. The mechanism is unknown, but is compatible with the release of an unknown bronchodilator by DI before the administration of Mch.

Whatever the cause of the apparent ineffectiveness of changes in lung volume to modulate airway smooth muscle tension in asthma, the lack of this important bronchodilating mechanism can explain the paradox of the dose-response curve in nonasthmatic subjects reaching a plateau with minimal changes in pulmonary function (31-33) in spite of complete closure of airways occurring at high transmural pressures in vitro $(16,17)$. Conditions associated with limited changes in lung volume might be expected to demonstrate increased airways responsiveness to bronchoconstrictors. For example, there is a recent report that in cervical spinal cord injury, where there is a reduction in FVC and inspiratory capacity, there is a marked increase in responsiveness to Mch (34).

In conclusion, we have demonstrated that the bronchodilating effect of DI is a major feature differentiating the normal from the asthmatic state. DI does not appear to have significant effects on the asthmatic airway. In the presence of DI, nonasthmatics are resistant to bronchoconstriction; in the absence of DI, bronchoconstriction can occur in the nonasthmatics to the same extent as in the asthmatics. We propose that this difference in response to DI is the major contributor to the hyperresponsiveness in asthma. The reason for the marked difference in the response to DI between normal and asthmatic subjects is not clear.

\section{Acknowledgments}

The authors would like to thank Ms. Brenda L. Jordan for her excellent assistance in the preparation of this manuscript.

This work was supported by National Institutes of Health grant P0 HL-49545.

\section{References}

1. Nadel, J. A., and D. F. Tierney. 1961. Effect of a previous deep inspiration on airway resistance in man. J. Appl. Physiol. 16:717-719.

2. Fish, J. E., M. G. Ankin, J. F. Kelly, and V. I. Peterman. 1981. Regulation of bronchomotor tone by lung inflation in asthmatic and nonasthmatic subjects. J. Appl. Physiol. Respir. Environ. Exercise Physiol. 50:1079-1086.

3. Sasaki, H., and F. G. Hoppin, Jr. 1979. Hysteresis of contracted airway smooth muscle. J. Appl. Physiol. Respir. Environ. Exercise Physiol. 47:12511262.

4. Parham, W. M., R. H. Shepard, P. S. Norman, and J. E. Fish. 1983. Analysis of time course and magnitude of lung inflation effects on airway tone: relation to airway reactivity. Am. Rev. Respir. Dis. 28:240-245.

5. Colebatch, H. J. H., K. E. Finucane, and M. M. Smith. 1973. Pulmonary 
conductance and elastic recoil relationships in asthma and emphysema. J. Appl. Physiol. 34:143-153.

6. Green, M., and J. Mead. Time dependence of flow-volume curves. 1974 J. Appl. Physiol. 37:793-797.

7. American Thoracic Society. 1962. Chronic bronchitis, asthma and pulmonary emphysema. Am. Rev. Respir. Dis. 84:762-768.

8. Permutt, S., and H. A. Menkes. 1978. Spirometry: analysis of forced expiration within the time domain. In The Lung in the Transition between Health and Disease. Vol 12. P. T. Macklem and S. Permutt, editors. Marcel Dekker, Inc., New York. 113-152.

9. Miller, A. 1986. Pulmonary Function Tests in Clinical and Occupational Lung Disease. Grune \& Stratton, Inc. Orlando, FL. 27-29.

10. Lapp, N. L., and R. E. Hyatt. 1967. Some factors affecting the relationship of maximal expiratory flow to lung volume in health and disease. Dis. Chest. 51:475-481.

11. Pride, N. B., S. Permutt, R. L. Riley, and B. Bromberger-Barnea. 1967. Determinants of maximal expiratory flow from the lungs. J. Appl. Physiol. 23:646-662.

12. Kirby, J. B., E. F. Juniper, F. E. Hargreave, and N. Zamel. 1986. Total lung capacity does not change during methacholine-stimulated airway narrowing. J. Appl. Physiol. 61:2144-2147.

13. Pellegrino, R., V. Benedetto, R. Selleri, and V. Brusasco. 1994. Changes in residual volume during induced bronchoconstriction in healthy and asthmatic subjects. Am. J. Respir. Crit. Care Med. 150:363-368.

14. Wheatley, J. R., P. Paré, and L. A. Engel. 1989. Reversibility of induced bronchoconstriction by deep inspiration in asthmatic and normal subjects. Eur. Respir. J. 2:331-339.

15. Ding, D. J., J. G. Martin, and P. T. Macklem. 1987. Effects of lung volume on maximal methacholine-induced bronchoconstriction in normal humans. $J$. Appl. Physiol. 62(3):1324-1330.

16. Murtagh, P. S., D. F. Proctor, S. Permutt, B. Kelly, and S. Evering. 1971 Bronchial closure with Mecholyl in excised dog lobes. J. Appl. Physiol. 31:409415.

17. Gunst, S. J., and J. Q. Stropp. 1988. Pressure-volume and length-stress relationships in canine bronchi in vitro. J. Appl. Physiol. 64:2522-2531.

18. Gunst, S. J., J. Q. Stropp, and J. Service. 1990. Mechanical modulation of pressure-volume characteristics of contracted canine airways in vitro. J. Appl. Physiol. 68:2223-2229.

19. Gunst, S. J. 1983. Contractile force of canine airway smooth muscle during cyclical length changes. J. Appl. Physiol. Respir. Environ. Exercise Physiol. 55:759-769.

20. Reid, L. M., G. J. Gleich, J. Hogg, J. Kleinerman, and L. A. Laitinen.
1989. Pathology. In The Role of Inflammatory Process in Airway Hyperresponsiveness. S. T. Holgate, editor. Blackwell Scientific Publications Ltd., Oxford. 36-79.

21. Mead, J., T. Takishima, and D. Leith. 1970. Stress distribution in lungs: a model of pulmonary elasticity. J. Appl. Physiol. 28:596-608.

22. Lai-Fook, S. J., R. E. Hyatt, and J. R. Rodarte. 1978. Effect of parenchyma shear modulus and lung volume on bronchial pressure-diameter behavior. J. Appl. Physiol. 44:859-868.

23. Gunst, S. J., D. O. Warner, T. A. Wilson, and R. E. Hyatt. 1988. Parenchymal interdependence and airway response to methacholine in excised dog lobes. J. Appl. Physiol. 65:2490-2497.

24. Lai-Fook, S. J., K. C. Beck, J. T. Donaldson, and A. M. Sutcliff. 1984 Effect of edema and height on bronchial diameter and shapes in excised dog lung. Respir. Physiol. 55:223-237.

25. Macklem, P. T. 1989. Mechanical factors determining maximum bronchoconstriction. Eur. Respir. J. 2(Suppl 6):516S-519S.

26. Burns, C. B., W. Taylor, and R. H. Ingram, Jr. 1985. Effects of deep inhalation in asthma: relative airway and parenchymal hysteresis. J. Appl. Physiol. 59:1590-1596.

27. Pliss, L. B., E. P. Ingenito, R. H. Ingram, Jr. 1989. Responsiveness, inflammation, and effects of deep breaths on obstruction in mild asthma. J. Appl. Physiol. 66(5):2298-2304

28. Marthan, R., and A. J. Woolcock. 1989. Is a myogenic response involved in deep inspiration-induced bronchoconstriction in asthmatics? Am. Rev. Respir Dis. 140:1354-1358.

29. Pellegrino, R., B. Violante, E. Crimi, and V. Brusasco. 1991. Time course and calcium dependence of sustained bronchoconstriction induced by deep inhalation in asthma. Am. Rev. Respir. Dis. 144:1262-1266.

30. Malmberg, P., K. Larsson, B. M. Sundblad, and W. Zhiping. 1993. Importance of the time interval between $\mathrm{FEV}_{1}$ measurements in a methacholine provocation test. Eur. Respir. J. 6:680-686.

31. Michoud, M. C . J. Leloires, and R. Amyot. 1981. Factors modulating the inter-individual variability of airway responsiveness to histamine: the influence of $\mathrm{H} 1$ and $\mathrm{H} 2$ receptors. Bull. Eur. Physiopathol. Respir. 17:807-821.

32. Woolcock, A. J., C. M. Salome, and Y. Yan. 1984. The slope of the doseresponse curve to histamine in asthmatic and normal subjects. Am. Rev. Respir. Dis. 130:71-75.

33. Sterk, P. J., E. E. Daniel, N. Zamel, and F. I. Hargreave. 1985. Limited bronchoconstriction to methacholine using partial flow-volume curves in non asthmatic subjects. Am. Rev. Respir. Dis. 132:272-277.

34. Dicpinigaitis, P. V., A. M. Spungen, W. A. Bauman, A. Absgarten, and P. A. Almenoff. 1994. Bronchial hyperresponsiveness after cervical spinal cord injury. Chest. 105:1073-1076. 